\title{
A bacterial small RNA regulates the adaptation of Helicobacter pylori to the host environment
}

\author{
Ryo Kinoshita-Daitoku,2, Kotaro Kiga², Masatoshi Miyakoshi (1) ${ }^{3}$, Ryota Otsubo ${ }^{1,12}$, Yoshitoshi Ogura ${ }^{4,5}$, \\ Takahito Sanada,2, Zhu Bo ${ }^{2}$, Tuan Vo Phuoc 6,7, Tokuju Okano ${ }^{8}$, Tamako lida², Rui Yokomori ${ }^{9}$, Eisuke Kuroda,2, \\ Sayaka Hirukawa², Mototsugu Tanaka2,8,10, Arpana Sood ${ }^{2}$, Phawinee Subsomwong ${ }^{1}$, Hiroshi Ashida 8 , \\ Tran Thanh Binh ${ }^{6,7}$, Lam Tung Nguyen ${ }^{6}$, Khien Vu Van ${ }^{11}$, Dang Quy Dung Ho7, Kenta Nakai (1) 9 , \\ Toshihiko Suzuki ${ }^{8}$, Yoshio Yamaoka (D) ${ }^{6}$, Tetsuya Hayashi ${ }^{4}$ \& Hitomi Mimuro (D) ${ }^{1,2 \times}$
}

Long-term infection of the stomach with Helicobacter pylori can cause gastric cancer. However, the mechanisms by which the bacteria adapt to the stomach environment are poorly understood. Here, we show that a small non-coding RNA of H. pylori (HPnc4160, also known as IsoB or NikS) regulates the pathogen's adaptation to the host environment as well as bacterial oncoprotein production. In a rodent model of $H$. pylori infection, the genomes of bacteria isolated from the stomach possess an increased number of T-repeats upstream of the HPnc4160-coding region, and this leads to reduced HPnc4160 expression. We use RNAseq and ITRAQ analyses to identify eight targets of HPnc4160, including genes encoding outer membrane proteins and oncoprotein CagA. Mutant strains with HPnc4160 deficiency display increased colonization ability of the mouse stomach, in comparison with the wild-type strain. Furthermore, HPnc4160 expression is lower in clinical isolates from gastric cancer patients than in isolates derived from non-cancer patients, while the expression of HPnc4160's targets is higher in the isolates from gastric cancer patients. Therefore, the small RNA HPnc4160 regulates $H$. pylori adaptation to the host environment and, potentially, gastric carcinogenesis.

\footnotetext{
${ }^{1}$ Department of Infection Microbiology, Research Institute for Microbial Diseases, Osaka University, Osaka, Japan. ${ }^{2}$ Division of Bacteriology, Department of Infectious Diseases Control, International Research Center for Infectious Diseases, The Institute of Medical Science, The University of Tokyo, Tokyo, Japan. ${ }^{3}$ Division of Biomedical Science, Faculty of Medicine, University of Tsukuba, Tsukuba, Japan. ${ }^{4}$ Department of Bacteriology, Graduate School of Medical Sciences, Kyushu University, Fukuoka, Japan. ${ }^{5}$ Division of Microbiology, Department of Infectious Medicine, Kurume University School of Medicine, Kurume Fukuoka, Japan. ${ }^{6}$ Department of Environmental and Preventive Medicine, Faculty of Medicine, Oita University, Yufu Oita, Japan. ${ }^{7}$ Department of Endoscopy, Cho Ray Hospital, Ho Chi Minh, Vietnam. ${ }^{8}$ Department of Bacterial Pathogenesis, Infection and Host Response, Graduate School of Medical and Dental Sciences, Tokyo Medical and Dental University, Tokyo, Japan. ${ }^{9}$ Human Genome Center, The Institute of Medical Science, The University of Tokyo, Tokyo, Japan. ${ }^{10}$ Division of Nephrology and Endocrinology, The University of Tokyo School of Medicine, Tokyo, Japan. ${ }^{11}$ Department of GI Endoscopy, 108 Central Hospital, Hanoi, Vietnam. ${ }^{12}$ Present address: Laboratory of Pharmaceutical Integrated Omics, Department of Pharmaceutical Engineering, Facility of Engineering, Toyama Prefectural University, Toyama, Japan.『email: mimuro@biken.osaka-u.ac.jp
} 
T he gastric pathogen Helicobacter pylori infects approximately half of the world's population and increases the risk of developing peptic ulcers, chronic gastritis, intestinal metaplasia, and gastric cancer ${ }^{1-3}$. The genomic diversity characteristic of $H$. pylori is crucial for its establishment of persistent infections in hosts and confers its adaptability to extreme gastric environments ${ }^{4,5}$. A well-known example of $H$. pylori's strong diversity is the presence of outer membrane proteins (OMPs), which are highly antigenic cell surface proteins and are thought to allow $H$. pylori to escape from host immunity during chronic infection $^{6}$. Mutations in OMP genes are characterized by simple repetitive sequences such as mononucleotide repeats (e.g., poly-T) and dinucleotide repeats (e.g., CT-repeats) ${ }^{6}$. The expansion and contraction of these simple repetitive sequences result in a genetically heterogeneous bacterial population, with phase variations controlled by the ON/OFF gene expression of the proteins $s^{4,6-8}$. However, whether the $H$. pylori adaptation to the host is controlled only by the diversity of mRNA containing simple repetitive sequences remains unclear. Because of the prevalence of small regulatory RNAs (sRNAs) in $\mathrm{H}$. pylori ${ }^{9}$, it is also possible that a heterogeneously mutated sRNA changes the expression levels of its target mRNAs or a mutation in the target sequence results in dysregulation by an $\mathrm{sRNA}^{10}$. Therefore, to understand the adaptation mechanisms of $H$. pylori, we analyzed bacterial gene mutations acquired by $H$. pylori over the course of infection using an experimental animal infection system in hosts of identical genetic background.

Here, we show that a small non-coding RNA HPnc4160 of H. pylori regulates the pathogen's adaptation to the host environment as well as bacterial oncoprotein production. We revealed that during the infection, a T-repeat length upstream of the coding region for HPnc4160 is elongated in H. pylori and that this elongation leads to decreased expression of HPnc4160. Through RNA-seq and iTRAQ analysis we identified several targets of HPnc4160, including cagA, a known carcinogenic protein. Importantly, we found that HPnc4160 expression is lower in clinical isolates from gastric cancer patients than in isolates derived from non-cancer patients, while the expression of HPnc4160's targets is higher in the isolates from gastric cancer patients. Our findings demonstrate that the small RNA HPnc4160 regulates H. pylori adaptation to the host environment and, potentially, gastric carcinogenesis.

\section{Results}

Identification of mutations accumulated during infection. To analyze the bacterial gene mutations acquired by $H$. pylori during persistent infection, Mongolian gerbils $(n=10)$ were inoculated with the H. pylori ATCC 43504 wild-type strain for 8 weeks with stable gastric colonization ${ }^{11}$. H. pylori were isolated from the infected stomachs (4 clones per gerbil, total 40 clones; Fig. 1a) and then analyzed by comparative whole-genome sequencing (Supplementary Fig. 1a, Supplementary Data 1 and 2). By integrating the genomic positions of these mutations, we identified 13 regions (R1, R3-R5, R7-R8, R10-R16) in which mutations were introduced in $50 \%$ or more of the strains (Supplementary Fig. 1a, Supplementary Data 2).

To investigate whether these mutated regions affect gene expression, transcripts in the mutated regions were quantified in isolates obtained from gerbils. Among the 15 corresponding coding (CDS) and non-coding RNA sequences (HP0947, babA, tpiA, jhp1163, HP0811, HPnc4160, HPnc4170, jhp0540, araS, pldA, sabA, HP1354, hopZ, tlpB, and HPB8_818), the expression of HPnc4160 showed the greatest fluctuation (Fig. 1b, Supplementary Fig. 1a, b). Similar results were obtained from strains (2 clones per mouse, total 10 clones) isolated from C57BL/6 mice
( $n=5)$ infected with $H$. pylori ATCC 43504 wild-type strain for 8 weeks (Supplementary Fig. 1a-c; Supplementary Data 3 and 4).

HPnc4160 (IsoB) was previously proposed as a cis-acting sRNA for HPnc4170 (aapB small ORF homolog) (Supplementary Fig. 2a) $)^{9,12}$. HPnc4160 and its upstream T-repeat region are conserved in $H$. pylori strains, but the T-repeat length in the promoter region is highly variable (Supplementary Fig. 2b). One to four additional thymidine bases were inserted into the T-repeat of our isolates from rodents, with the repeat length increasing with duration of period (Fig. 1c, Supplementary Fig. 2c-f). However, expansion of this region was not observed in long-term in vitro culture of wild-type strain (Supplementary Fig. 2g). Next, we analyzed changes in HPnc4160 expression according to the Trepeat length. In strains recovered from the $H$. pylori-infected stomachs of gerbils, HPnc4160 expression decreased with the expansion of the T-repeat (Fig. 1d, e). To exclude the effects of mutations other than those of T-repeats in strains recovered from rodents, we further analyzed the RNA expression of HP0811, HPnc4170, and HPnc4160 in isogenic variants of ATCC 43504 wild-type strains harboring different T-repeat lengths at the promoter region of HPnc4160 (T0 to T20, Fig. 1f, g, Supplementary Fig. 3a-c). The expression of HPnc4160 with varying T-repeat lengths was multiphasic: low in T1, high in T4, high in T14 (number of repeats in ATCC 43504 wild-type), low in T16, and intermediate in T18 (Fig. 1f, g). Expression levels of hpnc4170 and its downstream HP0811 were not affected by the T-repeats (Supplementary Fig. 3b-c), indicating that HPnc4160 is unlikely to act as a cis-acting sRNA.

Identification of HPnc4160 target genes. Helicobacter pylori utilizes RNA-mediated regulation in trans through relatively short base-pairing with multiple mRNAs from different loci despite the absence of known RNA matchmakers such as Hfq and ProQ ${ }^{13,14}$. To identify the target mRNA of HPnc4160, we generated a $\Delta h p n c 4160-h p n c 4170$ strain, in which both HPnc4160 and HPnc4170 on the complementary strand were deleted. mRNA and protein expression analysis identified eight differentially expressed factors in the mutant strain compared to in the wild-type (cagA, hofC, HELPY_1262, HP0410, horB, omp14, hopE, and HP1227; $P<0.001$ by RNA-seq; $P<0.01$ by isobaric tag for relative and absolute quantitation labeling and liquid chromatography-tandem mass spectrometry analysis) (Fig. 2a-c, Supplementary Data 5 and 6). Notably, five of these eight factors (HofC, HP0410, HorB, Omp14, and HopE) were annotated as outer membrane proteins. More strikingly, the expression of cagA mRNA and protein, which is a known bacterial oncoprotein ${ }^{1}$, were most noticeably increased (Fig. 2a, b, Supplementary Data 5 and 6).

We analyzed whether the mRNA expression of these eight factors depended on the presence of HPnc4160. Levels of HPnc4160 trended to decrease, with T16 exhibiting the lowest value, whereas the mRNA of the eight candidates trended to increase, with T16 exhibiting the highest value (Fig. 1f, g; Supplementary Fig. 3a-d). The expression of these target mRNAs and the presence of HPnc4160 showed a strong inverse correlation (Spearman correlation coefficient $(r)=-0.8234$ to -0.7312) (Supplementary Fig. 3d). The HPnc4160 overexpression strain (WT/pHel2-hpnc4160) showed significantly decreased expression of the target mRNAs. In contrast, in the $\Delta h p n c 4160$ hpnc4170 strain, the mRNA expression of each target increased compared to in the wild-type (Supplementary Fig. 3e-h). As the $\Delta h p n c 4160-h p n c 4170$ strain lacks the HPnc4170 sequence in the complementary strand of HPnc4160, we constructed a $\Delta h p n c 4160-h p n c 4170 / \mathrm{pHel2}-h p n c 4160$ strain complementing only HPnc4160 to confirm the effect of the HPnc4170 sequence on HPnc4160 target mRNA expression. Compared to the 
a

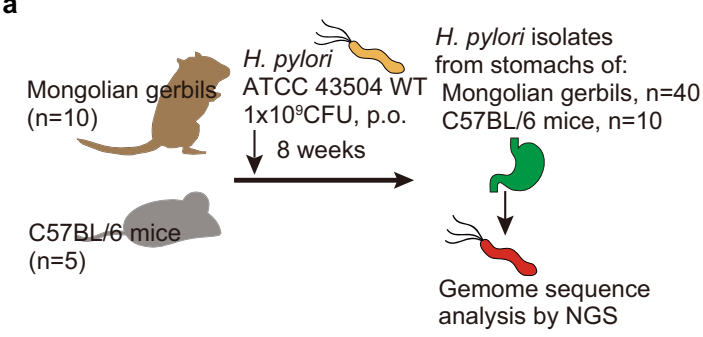

b Strains from Mongolian gerbils infected with ATCC 43504 WT

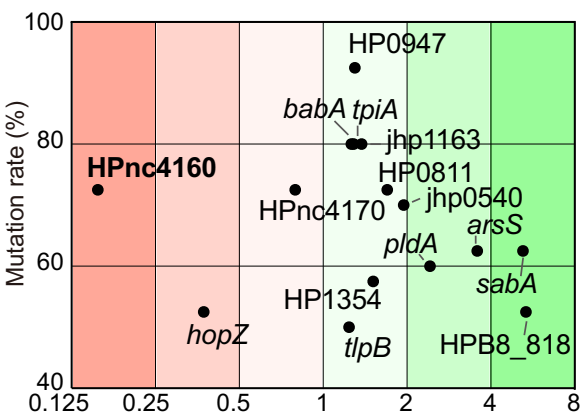

Fold change of RNA expression level [mut (+) / input]

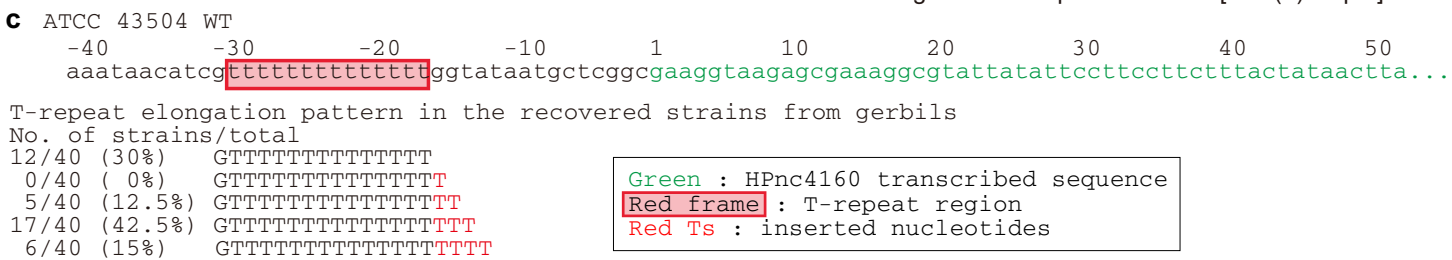

$6 / 40(15 \%) \quad$ GTTTTTTTTTTTTTTTmTT

d Strains from Mongolian gerbils infected with ATCC 43504 WT
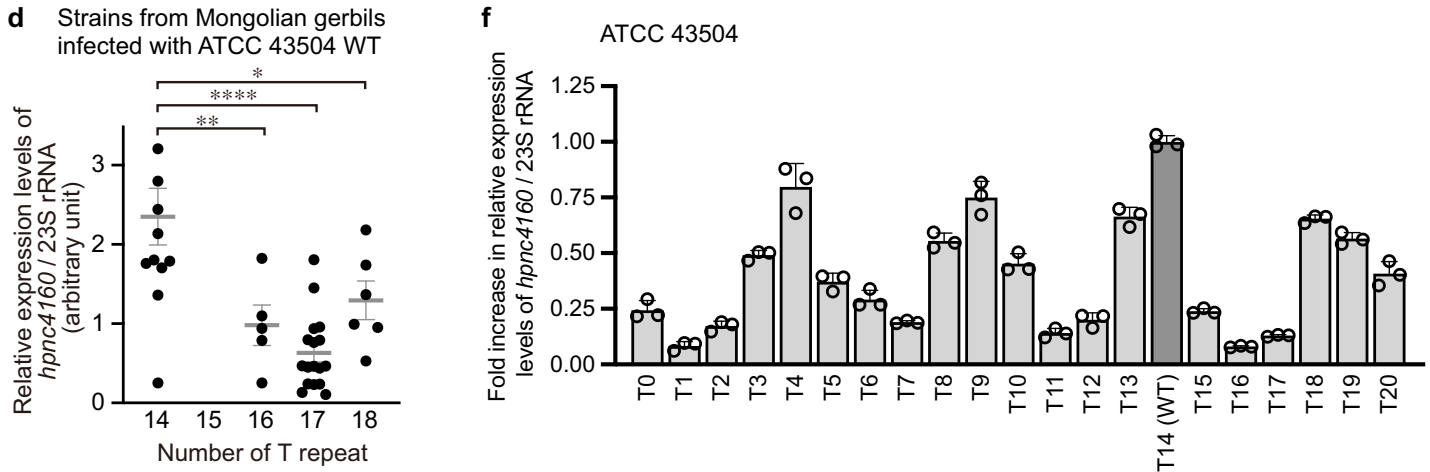

e

Strains from Mongolian gerbils infected with ATCC 43504 WT g

ATCC 43504

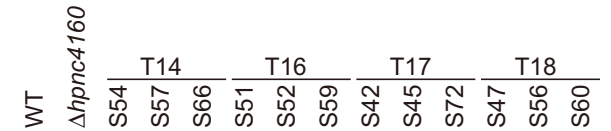
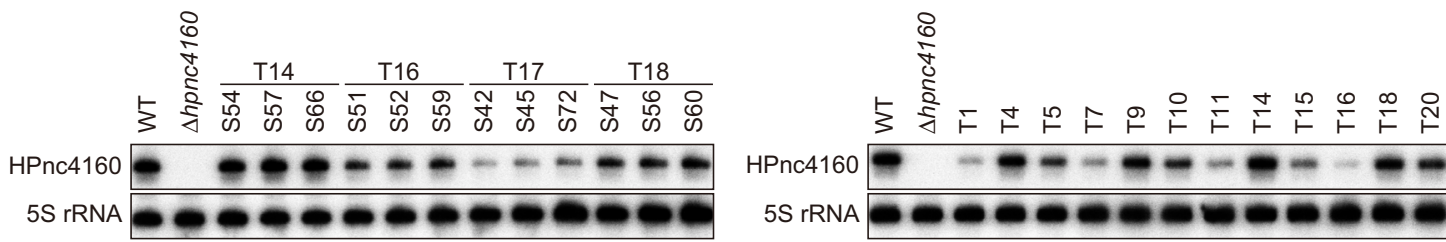

HPnc4160

5S rRNA

Fig. $1 \mathrm{H}$. pylori acquires T-repeat extension upstream of HPnc4160 to decrease its expression during infection in vivo. a Schematic showing experimental strategy. b RNA expression levels of ORFs or nearby genes of genome regions mutated in $>50 \%$ of the strains recovered from gerbils, plotted against mutation rates. c DNA sequence around the HPnc4160 and T-repeat sequence of the strains recovered from gerbils. $\mathbf{d}-\mathbf{g}$ Relative $h p n c 4160$ expression in $H$. pylori strains recovered from gerbils (T repeat number of $14, n=12 ; 16, n=5 ; 17, n=17 ; 18, n=6$ ) and genetically modified T-repeat lengths $(n=3)$ in RT-PCR $(\mathbf{d}, \mathbf{f})$ and Northern blot $(\mathbf{e}, \mathbf{g})$. Shown are the means \pm s.d $(\mathbf{d}, \mathbf{f})$. Data were processed by Grubbs' test for outliers. ${ }^{\star} P=0.0379$, ${ }^{\star \star} P=0.0088,{ }^{\star \star \star \star} P<0.0001$ by Sidak's multiple comparison tests (two sided) (d). Data are representative of two independent experiments (d-g). Source data are provided as a Source Data file.

$\Delta$ hpnc4160-hpnc4170 strain, the mRNA expression levels of the candidates were decreased in the HPnc4160 complemented $\Delta h p n c 4160-h p n c 4170$ /pHel2-hpnc4160 strain (Supplementary Fig. 3h). These data indicate that the eight target candidates are negatively regulated by HPnc4160.

Trans-acting sRNAs generally form base-pairing in the $5^{\prime}$ untranslated region (UTR) or CDS of target mRNAs to repress or activate gene expression at the post-transcriptional level ${ }^{15,16}$. In H. pylori, the best-characterized sRNA RepG (HPnc5490) binds to G-repeats in $5^{\prime} \mathrm{UTR}$ of $\operatorname{tlp} B$ mRNA to repress translation and destabilize $\mathrm{mRNA}^{9,10}$. As $H$. pylori lacks RNase E/G homologs 13,17 , which promote mRNA degradation by sRNAs, other endoribonuclease such as RNase III may degrade target mRNA accompanied by translation inhibition.
In the $5^{\prime} \mathrm{UTR}$ of seven identified target genes except cagA, we found a sequence that binds complementarily to the loop region of the HPnc4160. (Supplementary Fig. 4a, b). The direct binding of HPnc4160 to the $5^{\prime}$ UTR of the seven target genes except cagA were confirmed by Electrophoretic mobility shift assays (Fig. 3a). Instead, we identified five putative target sites in the CDS of cagA (Type 1 at $2344 \mathrm{nt}$ from the start codon, and Type 2 at four positions of 2838, 2940, 3042, and 3144 nt) (Fig. 3b-d). We confirmed the direct binding of partial cagA CDS (positions 2778-3236 nt from start codon of cagA) to HPnc4160 (Fig. 3e). This binding was abolished for Non-Binding (NB)-cagA RNA in which the four Type 2 HPnc4160 binding sequences were mutated and the amino acid sequence of CagA was preserved (Fig. 3b, e-g). The abolished binding of NB-cagA RNA was 

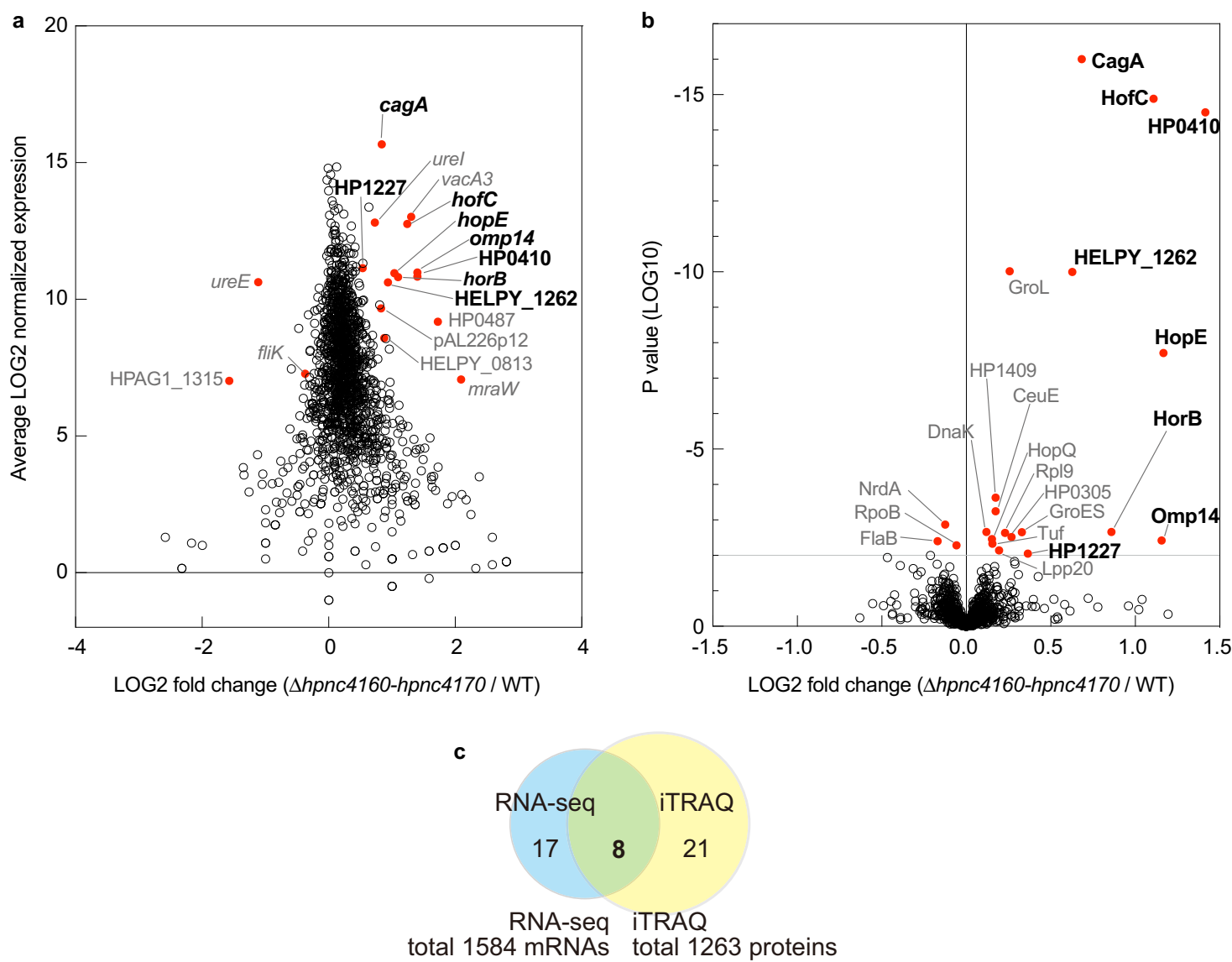

Fig. 2 HPnc4160 downregulates the expression of bacterial pathogenic factors. a MA plot of ratios $[\Delta h p n c 4160-h p n c 4170 /$ wild-type (WT) $H$. pylori] versus their normalized average mRNA expression determined by RNA-sequencing (RNA-seq). Red dots, $P<0.001$ by a Fisher Exact Test (two-sided). b Volcano plot of proteins quantified by isobaric tags for relative and absolute quantification analysis comparing WT and $\Delta h p n c 4160-h p n c 4170$. Each point represents the difference in protein expression (fold-change) between the two groups plotted against the level of statistical significance. Red dots, $P<0.01$ by the paired t-test (two-sided). c Venn diagram representing the number of significantly differentially expressed factors between $\Delta h p n c 4160$ - $h p n c 4170$ and WT. Source data are provided as a Source Data file.

restored by complementary mutations in HPnc4160 at positions 64, 67, and 73 (HPnc4160 mut; Fig. 3e, h), demonstrating direct base-pairing between HPnc4160 and cagA. In addition, we found that $H$. pylori RNase III recombinant protein degraded the dsRNA upon binding between HPnc4160 and biotin-labeled partial cagA mRNA, but not NB-cagA RNA (Fig. 3i, Supplementary Fig. 4c). These data indicate that HPnc4160 controls cagA at the post-transcriptional level by binding to multiple binding sequences present in its CDS region and possibly promoting the degradation of mRNA by RNase III.

Effects of HPnc4160 on $\boldsymbol{H}$. pylori pathogenicity. We further analyzed CagA, which has been shown to be deeply involved in pathogenesis ${ }^{1-3}$. The $H$. pylori expressing NB-cagA, in which all five HPnc4160-binding sequences were mutated but the amino acid sequence was preserved, showed significantly increased expression of cagA mRNA and protein to the same extent as that of the $\Delta h p n c 4160-h p n c 4170$ strain (Supplementary Fig. 5a-c). The increased expression of $\operatorname{cag} A$ mRNA and protein was negated by complementary mutations in $h p n c 4160$ ( $h p n c 4160$ mut) (Supplementary Fig. 5a-c), indicating that HPnc4160 negatively controls the expression of cagA mRNA and protein in $H$. pylori. H. pylori injects CagA proteins into the host epithelium via a Type IV secretion system (TFSS), and then intracellular CagA proteins are phosphorylated at tyrosine residues (pY) by the host
Src/Abl kinase ${ }^{18,19}$. Using pY-CagA-specific antibodies, we confirmed that intracellular CagA increased in NB-cagA-infected cells, accompanied by an increase in total CagA, but not in hpnc4160 mut strain-infected cells (Supplementary Fig. 5d). Intracellular CagA induces AGS cell motility (scattering/hummingbird phenotype $)^{20,21}$. More elongated AGS cells were observed during infection with $\Delta h p n c 4160-h p n c 4170$ or NB-cagA strains vs in the wild-type or $\Delta h p n c 4160-h p n c 4170 / \mathrm{pHel} 2$ hpnc4160 strain-infected cells (Supplementary Fig. 5e, f). The amount of IL-8 secreted from $H$. pylori-infected cells, which is primarily induced by intracellular $\mathrm{CagA}^{22}$, was higher in NB$\operatorname{cagA}$ strain-infected cells than in wild-type strain or hpnc4160 mut strain-infected cells (Supplementary Fig. 5g). These results suggest that the binding of HPnc4160 to cagA mRNA is critical for controlling the amount of functional CagA protein in H. pylori.

We further assessed changes in CagA activity dependent on $\mathrm{T}$ repeat length of $h p n c 4160$. Western blot analysis showed that 48 out of 50 strains recovered from rodents infected with ATCC 43504 at 8 weeks post infection expressed VirB7 (TFSS protein) and retained the ability to translocate CagA into host epithelium (Supplementary Fig. 5h, i). Intracellularly translocated CagA protein tends to be higher in strains with T-repeat numbers 16 and 17 with low levels of HPnc4160, and lower in T14 and T18 with high levels of HPnc4160. A similar trend of increased IL-8 expression with T-repeat length was observed in the expression of 
a
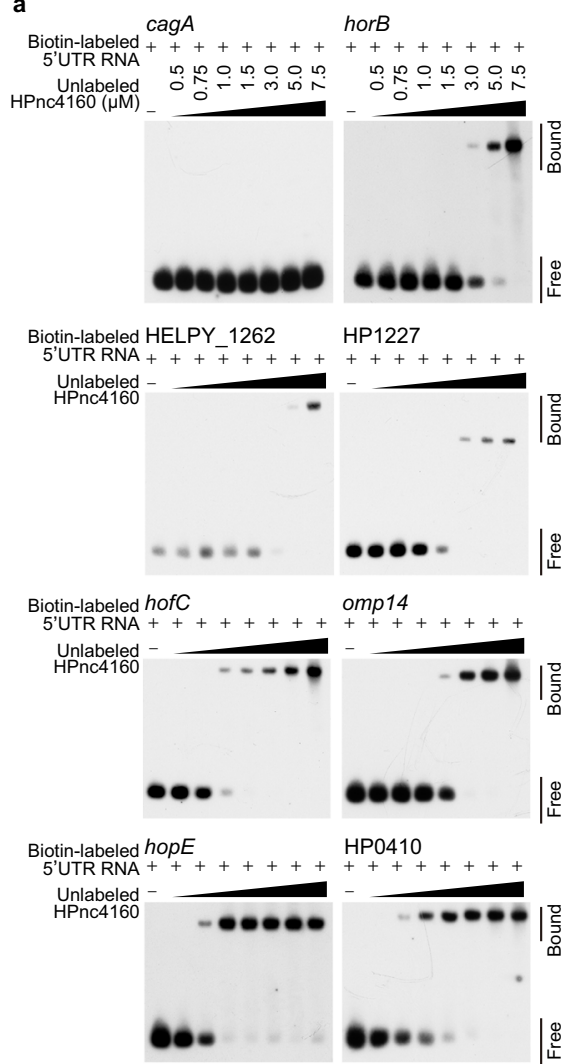

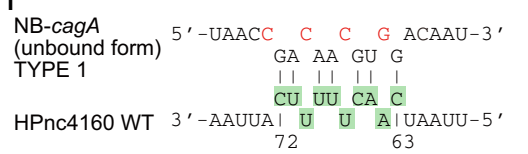

g 5'-GGCAC C C G ACGAU-3' TYPE 2 GA AA GU G

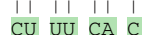
HPnc4160 WT 3'-AAUUA I U U A AIUAAUU-5

\section{h}

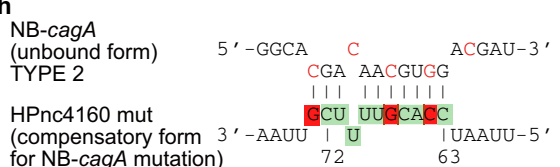

b

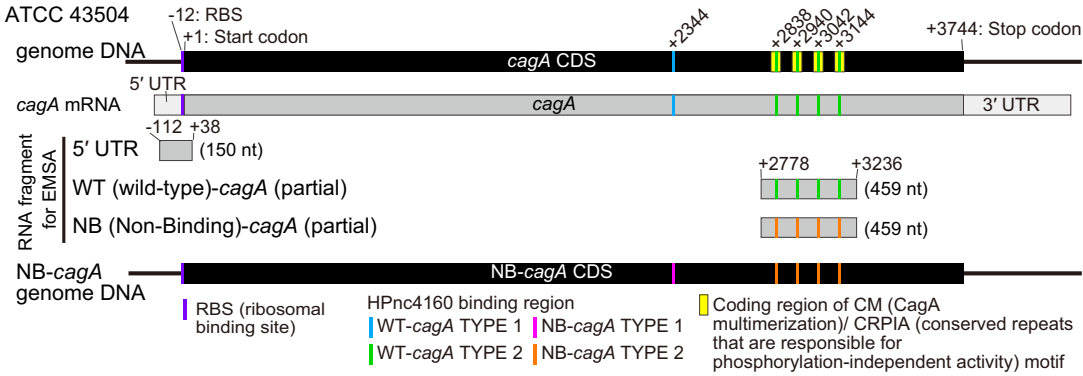

c WT-CagA $5,-U A A C G$ d

WT-cagA 5'-UAACG U ACAAU-3' WT-cagA 5'-GGCAU U AUGAU-3'

$\begin{array}{llll}\text { TYPE } 1 & \text { GA AAAGUUG } & \text { TYPE 2 AAAGUUG }\end{array}$

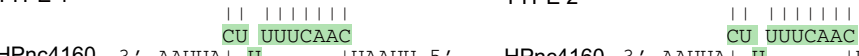

$\begin{array}{lcclcc}\text { HPnc4160 } & 3^{\prime} \text {-AAUUA I U } & \text { IUAAUU-5' } & \text { HPnc4160 } & 3^{\prime} \text {-AAUUA I U } & \text { IUAAUU-5 } \\ \text { WT } & 72 & 63 & \text { WT } & 72 & 63\end{array}$

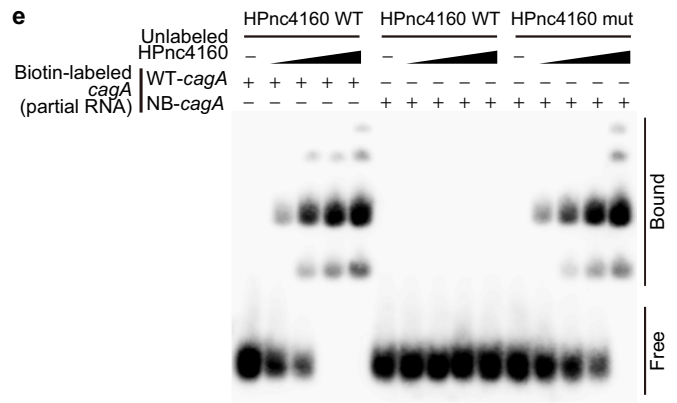

i

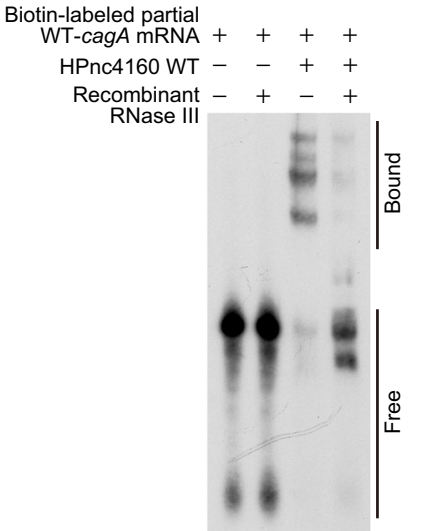

Fig. 3 HPnc4160 binds to target mRNA. a Electrophoretic mobility shift assay (EMSA) analysis of HPnc4160 binding to the $5^{\prime} U T R$ region of each candidate mRNA. Data are representative of two independent experiments. b Schematic of CagA motifs, HPnc4160 binding regions, and HPnc4160-nonbinding $\operatorname{cag} A(\mathrm{NB}-\operatorname{cag} A)$. c, d Schematic of predicted HPnc4160 binding sites in the corresponding CDS sequence of cagA TYPE 1 (c) and TYPE 2 (d). Upper sequences indicate target cagA mRNA sequences; lower sequences indicate the HPnc4160 sequence with base numbers. Colored sequences correspond to the loop structures indicated in Supplementary Fig. 4a. e EMSA analysis of HPnc4160 WT or HPnc4160 mut (a compensatory form of NBcagA) bindings to RNA of partial cagA WT or NB-cagA. Data are representative of two independent experiments. $\mathbf{f}-\mathbf{h}$ Schematic of CDS sequence of the NB-cagA of TYPE 1 (f) and TYPE 2 (g) and HPnc4160 mut (h). Upper sequences indicate target cagA mRNA sequences; lower sequences indicate the HPnc4160 sequence with base numbers. Green-colored sequences correspond to the loop structures of HPnc4160 indicated in Supplementary Fig. $4 a$. Mutated nucleotides in the cagA mRNA sequence are shown in red. Red-colored sequence correspond to mutated nucleotides in the hpnc4160 sequence (h). i RNase protection assay with HPnc4160, cagA mRNA, and recombinant RNase III. Data are representative of two independent experiments. Source data are provided as a Source Data file.

IL-8 in AGS cells infected with the T-repeat mutated strains (Supplementary Fig. 5j).

Next, to understand the significance of the HPnc4160 control mechanism in bacterial adaptation to the host, mice were orally inoculated with either wild-type, $\Delta h p n c 4160-h p n c 4170$, $\Delta h p n c 4160-h p n c 4170 / \mathrm{pHel} 2-h p n c 4160$, and NB-cagA, and the bacterial loads in the stomach were analyzed. At three days postinfection, a timepoint prior to T-repeat length extension (Supplementary Fig. 2e), levels of bacterial colonization and Cxcl2 mRNA in the stomach were found to be significantly increased for the $\Delta h p n c 4160-h p n c 4170$ strain compared to wildtype, whereas those of the $\Delta$ hpnc4160-hpnc4170/pHel2-hpnc4160 

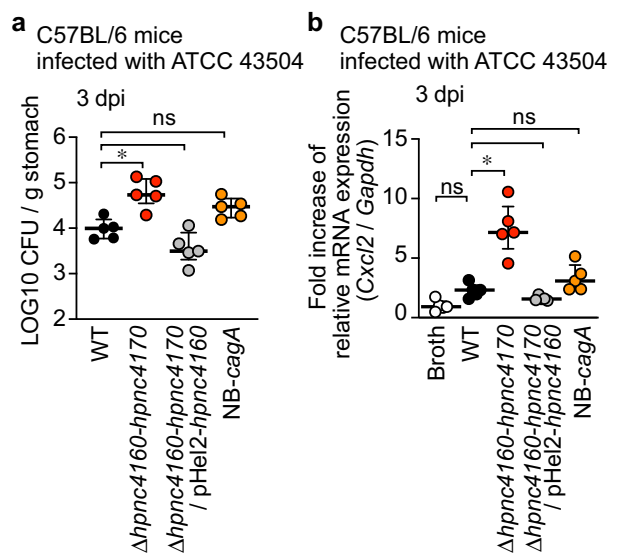

c $\quad$ C57BL/6 mice infected with ATCC 43504

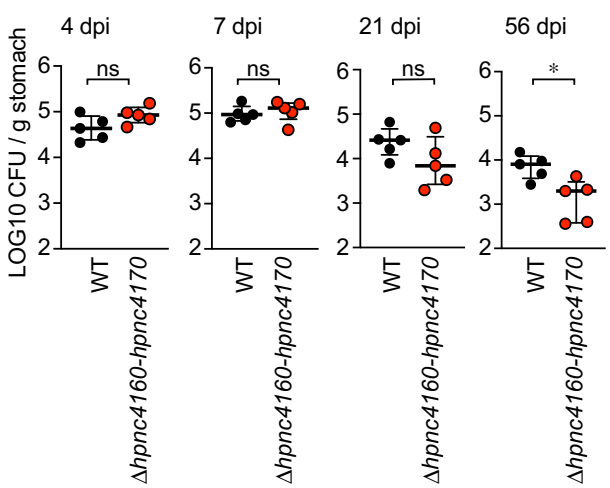

Fig. 4 HPnc4160 regulates bacterial-host adaptation and pathogenesis. a, b Three days after infection, quantitative culture assays (a) and RT-PCR (b) were performed on mouse gastric specimens. c Effect of HPnc4160 deletion of $\mathrm{H}$. pylori on bacterial load in mouse stomach at indicated days of post infection (dpi). Statistical significance determined by non-parametric Dunn's multiple comparison test (two-sided) (a, b) or two-tailed Mann-Whitney test (c). ${ }^{\star} P=0.0485(\mathbf{a}) ;{ }^{\star} P=0.0309(\mathbf{b}) ;{ }^{\star} P=0.0159$ (c); ns, not significant. Source data are provided as a Source Data file.

strain were equivalent to wild-type (Fig. 4a, b). Notably, both colonization levels and Cxcl2 mRNA levels were slightly higher following NB-cagA infection than following wild-type infection. This result indicates that factors controlled by HPnc4160, other than CagA, are important for bacterial adaptation and host gastritis development (Fig. 4a, b). Importantly, the bacterial colonization levels at 8 weeks post infection was significantly decreased in the $\Delta h p n c 4160$ - $h p n c 4170$-infected animals, as compared to the wild-type infection (Fig. 4c), indicating that HPnc4160 is beneficial for long-term colonization of H. pylori.

Effects of T-repeats length diversity on adaptation. Since $H$. pylori is known to be transmitted from human to human, we conducted reinfection experiments using isolates from mice with different T-repeats (T16 in Hp6 and T18 in Hp8) with equivalent in vitro growth rates (Supplementary Fig. 6a). Similar to the wildtype strain (Fig. 4a, c), both strains used for reinfection showed almost the same number of colonized bacteria in the stomachs from 3 days to 8 weeks after infection (Supplementary Fig. 6b). Interestingly, the strains used for reinfection displayed increased repeat lengths with increases in infection period, similar to the wild-type strain (Supplementary Figs. 2e and 6c). Furthermore, the strains with longer T-repeats (T16 and T18) were more likely to acquire the T-repeat elongation and adapt to the mice stomach than the strains with shorter T-repeats (T1 and T4) (Fig. 5a, b). These data indicate that repetitive sequence length variation of the T-repeat is advantageous for $H$. pylori persistent infection.

T-repeat length and HPnc4160 expression in clinical isolates. Because one of the HPnc4160 target factors was CagA, which is known as the strongest risk factor for $H$. pylori-related gastric cancer, we examined the expression of HPnc4160 target genes in clinical isolates from non-cancer patients and patients with cancer. Sequence analysis of clinical isolates showed that T-repeat regions were longer in strains derived from patients with cancer than in those derived from non-cancer patient (Fig. 6a, Supplementary Fig. 7, Supplementary Data 7). As shown in Fig. 6b, isolates from patients with cancer had lower levels of hpnc4160 but increased expression of six factors controlled by HPnc4160 (cagA, horB, hopE, omp14, hofC, and HP0410) compared to isolates from non-stomach cancer patients (Fig. 6b, Supplementary Fig. 7b, c). The relevance of HPnc4160 and H. pylori-related gastric cancer risk was also assessed by in vitro infection experiments. AGS cells infected with $H$. pylori expressing low levels of HPnc4160 displayed increased expression of CDX2, an indicator of intestinal epithelialization in the precancerous state (Supplementary Fig. 7d). In summary, our mutational analysis studies revealed a functional small RNA that regulates the pathogenicity of $H$. pylori and may have implications for gastric cancer development.

\section{Discussion}

We have demonstrated that mRNA expression of CagA and OMPs is suppressed by HPnc4160 at the onset of $H$. pylori infection and that over the course of infection, thymidine repeats are inserted into the upstream region of $h p n c 4160$, decreasing HPnc4160 expression, resulting in increased target mRNA expression; these factors contribute to bacterial adaptation to the host environment and potentiate gastritis and gastric oncogenesis (Fig. 7). Control of gene expression by varying the number of repeat sequences is a known control mechanism employed by $H$. pylori $^{23}$. The present study suggests that repeat sequences of the H. pylori genome are important, not only as an ON/OFF mechanism for protein expression, including that of cell adhesion factors SabA and $\mathrm{BabA}^{24}$, but also in sRNA expression. Since Trepeat is reported to affect the local DNA structure for RNA polymerase binding, by shifting the axial distance between the core promoter and upstream promoter elements, we speculate that steric distance between the promoter and the HPnc4160 transcription initiation site fluctuates as the T-repeat length increases or decreases, thereby modulating HPnc4160 expression $^{12,25}$. As variations in the T-repeat length upstream of hpnc4160 in H. pylori were observed in vivo (Figs. 1c and 5b; Supplementary Figs. 2c-f and 6c), through slipped strand mispairings ${ }^{26}$, the $H$. pylori population may have become genetically heterogeneous during the course of infection, ensuring that a bacterium suitable for host colonization was selected and propagated. Importantly, the repetitive sequence length variation is advantageous for prolonged infection of $H$. pylori (Fig. 5a). In this study, we primarily used the H. pylori strain ATCC 43504, a clinical isolate originating from the human antrum. The T-repeat length did not fluctuate during in vitro subculture of ATCC 43504 , but was found to increase upon isolation from infected rodents and ranged from 14-19 of T-repeat (Fig. 1c; Supplementary Fig. 2c-g), indicating that slipped strand mispairings were induced by stress conditions exerted by the host.

The HPnc4160-binding sequence appears five times in the ATCC $43504 \operatorname{cagA}$ CDS within the CM/CRPIA motifs in the CagA C-terminal region, which is known to bind with host signal 
a C57BL/6 mice infected with ATCC 43504

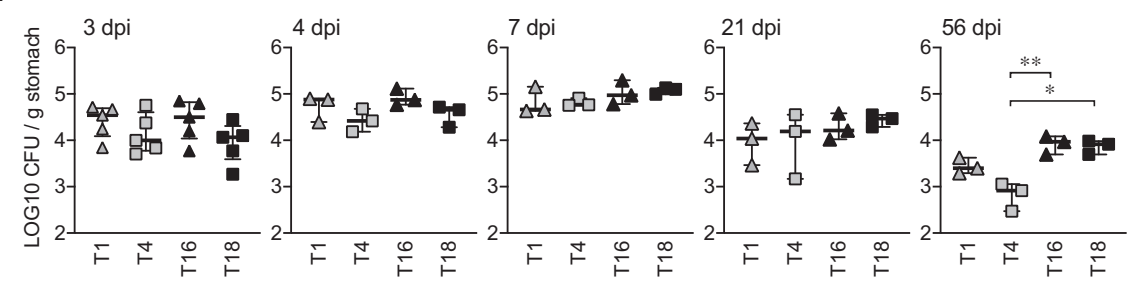

b $\quad$ C57BL/6 mice infected with ATCC 43504
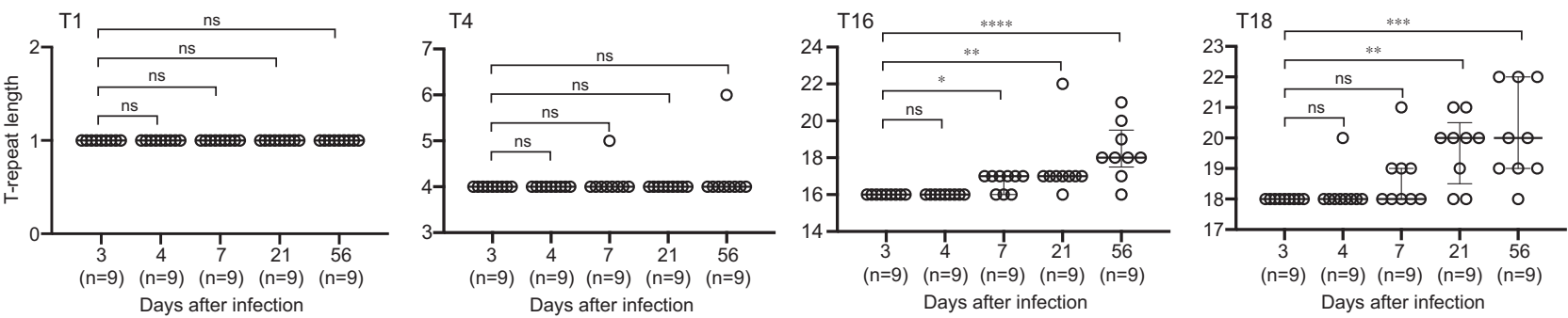

Fig. 5 Time-dependent changes in bacterial-host adaptation and the T-repeat length. $\mathbf{a}, \mathbf{b}$ Time-dependent changes in the bacterial load in the infected mouse stomach (a) and length of the T-repeat region (b) in T1, T4, T16 and T18 strains recovered from mice. Data are presented as the median with interquartile range. Statistical significance determined by uncollected Dunn's multiple comparison test (two-sided). ${ }^{\star} P=0.0174,{ }^{\star}{ }^{*} P=0.0066$ (a); $P=$ $0.0294,{ }^{\star \star} P=0.0014,{ }^{\star \star \star \star} P<0.0001(\mathbf{b}, \mathrm{T} 16) ;{ }^{\star \star} P=0.0012,{ }^{\star \star \star} P=0.0002(\mathbf{b}, \mathrm{T} 18)$; ns, not significant. Source data are provided as a Source Data file.

$\mathbf{a}$

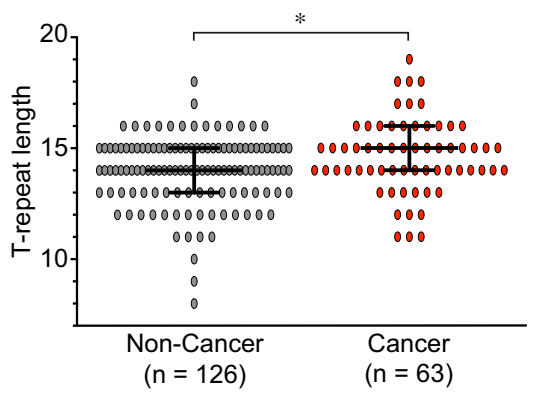

b
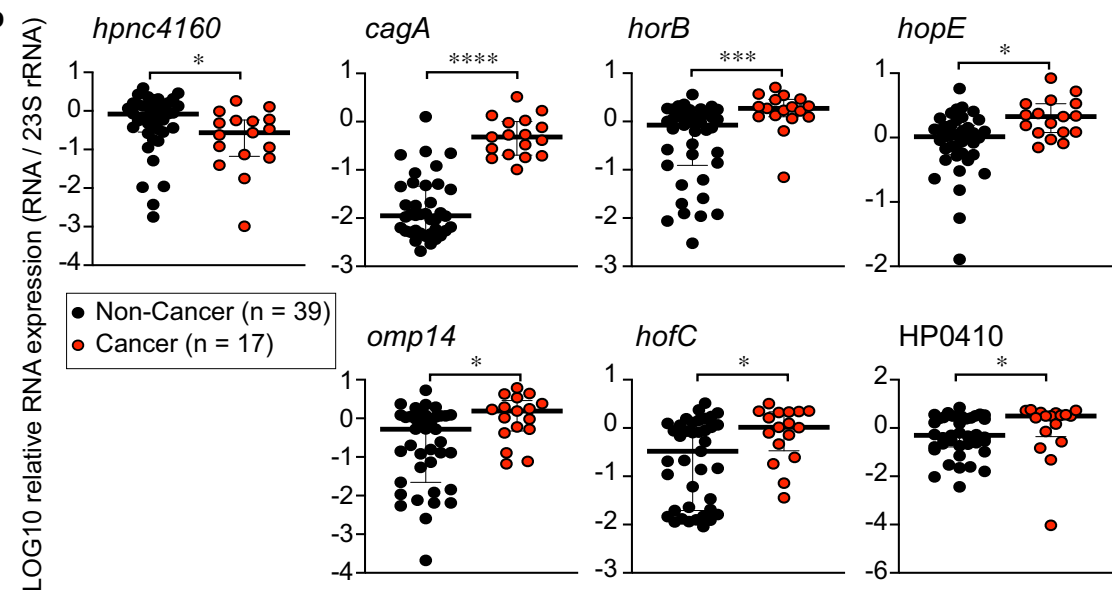

Fig. 6 T-repeat length and HPnc4160 expression in clinical isolates. a, b T-repeat length upstream of HPnc4160 (a) and expression of indicated mRNAs (b) in clinical isolates from non-cancer (non-cancer) and patients with cancer (cancer). Data are presented as means with $95 \%$ confidence interval (a) or medians with interquartile range (b). Statistical significance determined by two-tailed Mann-Whitney test (a, b). ${ }^{\star} P=0.0157$ (a); ${ }^{\star} P=0.0215$ ( $\left.h p n c 4160\right)$, ${ }^{\star \star \star \star} P<0.0001(\operatorname{cag} A)$, ${ }^{\star \star \star} P=0.0004$ (horB), ${ }^{\star} P=0.0025$ (hopE), ${ }^{\star} P=0.0110$ (omp14), ${ }^{\star} P=0.0122$ (hofC), ${ }^{\star} P=0.0205$ (HP0410) (b). Source data are provided as a Source Data file. 
in vitro

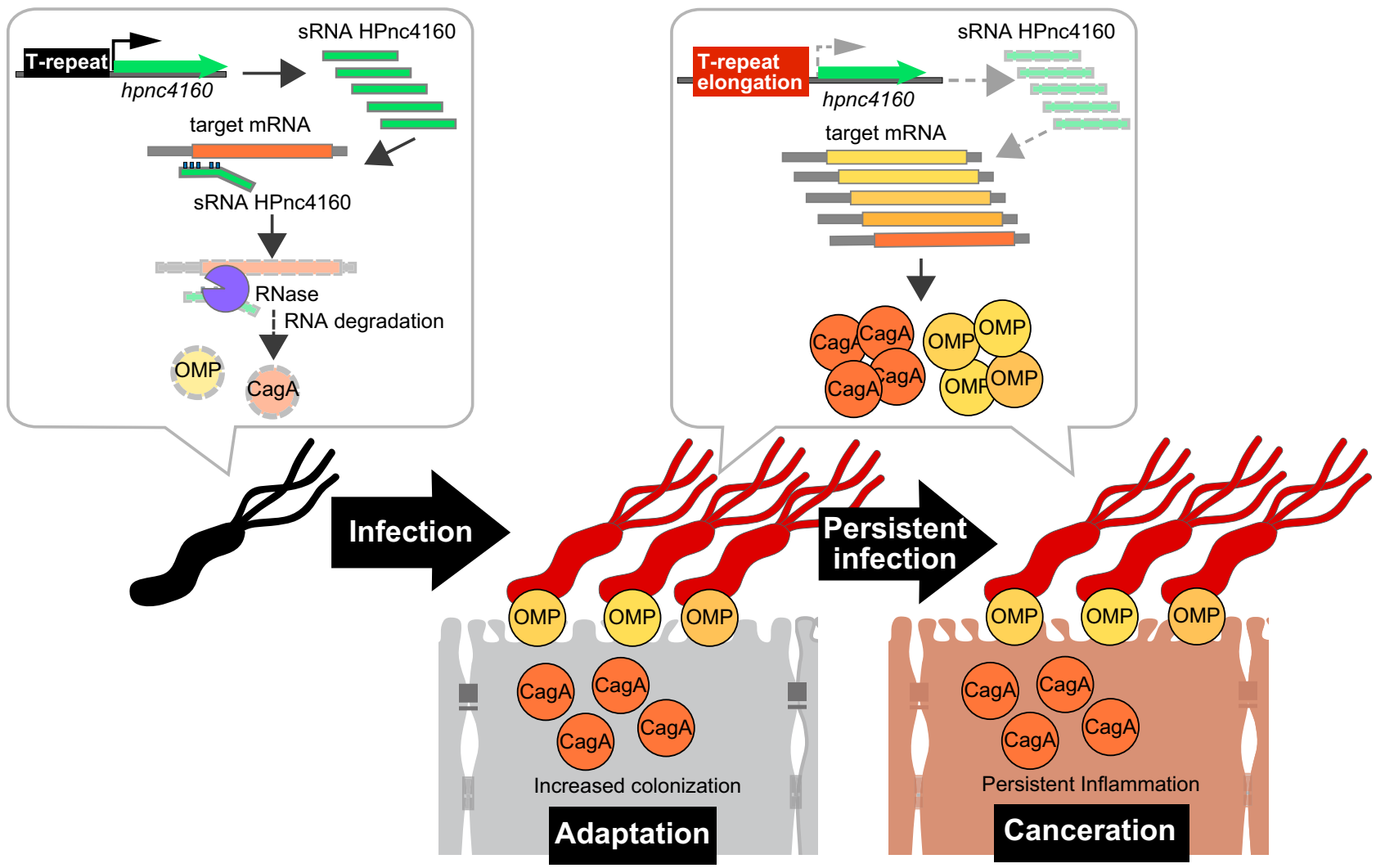

Fig. 7 Infection-induced silencing of HPnc4160 upregulates target gene expression and promotes bacterial-host adaptation and, potentially, oncogenesis during chronic $\boldsymbol{H}$. pylori infection. H. pylori infection in vivo leads to elongation of T-repeats upstream of the HPnc4160 sRNA coding region, which results in decreased expression of HPnc4160 sRNA. Gene silencing of HPnc4160 results in increased target gene expression, coding for OMPs and CagA. As a result, bacterial colonization and CagA translocation into the attached host cells increase, promoting bacterial adaptation to the host and, possibly, oncogenesis.

proteins $^{27,28}$ (Fig. 3b). Generally, regulation of mRNA expression by sRNA is induced by binding at one site. Multiple binding sequences in the cagA CDS may facilitate efficient reduction of cagA transcript levels in response to HPnc4160. While the present manuscript was under revision, Eisenbart et al. published a paper describing that the nickel-responsive transcriptional regulator NikR regulates the expression of HPnc4160 (which the authors of that study renamed as 'NikS'), and HPnc4160 represses multiple major virulence genes, including cagA and $v a c A$ in vitro ${ }^{29}$. They showed that HPnc4160 binds to the $5^{\prime} \mathrm{UTR}$ of the cagA mRNA of strain G27. Although we could not observe HPnc4160 binding to the $5^{\prime} \mathrm{UTR}$ region of the ATCC $43504 \operatorname{cagA}$ (Fig. 3a), they also identified a group of HPnc4160-regulating pathogenic factors that overlapped with those we found. Therefore, their report is in line with our finding that the sRNA HPnc4160 is a master regulator of Helicobacter pathogenicity.

All identified HPnc4160 targets encode large proteins that require greater energy expenditure for production (Fig. 2a-b, Supplementary Data 5 and 6). As the growth of bacterial cells in vitro may not require these pathogenic factors, hpnc4160 expression may be higher whereas its targets are suppressed in vitro. Upon entering the host stomach, $H$. pylori may activate a mechanism that decreases HPnc4160 production, thereby simultaneously increasing expression of OMPs and CagA, allowing the bacteria to efficiently adapt to environmental changes and colonize the gastric mucosa.

Because CagA is a carcinogenic protein related to gastric cancer, an increase in CagA levels achieved by down-regulation of
HPnc4160 may correlate with an increased risk of gastric cancer. Additionally, we demonstrated that several OMPs are also regulated by HPnc4160. These targets are likely involved in host adaptation, given that the HPnc4160 knockout strains display increased bacterial colonization and inflammatory cytokine expression, phenotypes not solely dependent on CagA production (Fig. 4a, b). Collectively, we find HPnc4160 behaves as a master regulator of $H$. pylori host adaptation and may serve to potentiate gastric carcinogenesis.

\section{Methods}

Data reporting. No statistical methods were used to predetermine sample size, the experiments were not randomized, and the investigators were not blinded to allocation during experiments and outcome assessment.

Strains and culture conditions. The isogenic mutants $\triangle c a g A$ and $\triangle v i r B 7$ of Helicobacter pylori strain ATCC 43504 were constructed in our laboratory ${ }^{11}$. The strain PMSS1 was generously provided by Dr, Manuel R. Amieva (Stanford University $)^{30}$. H. pylori was cultured on Trypticase soy agar with $5 \%(\mathrm{v} / \mathrm{v})$ sheep's blood (Thermo Fisher Scientific, Waltham, MA, USA) for 2 days at $37^{\circ} \mathrm{C}$ in microaerobic conditions. Bacterial colonies were suspended in Brucella broth (Thermo Fisher Scientific) supplemented with 5\% (v/v) inactivated fetal bovine serum (FBS; Thermo Fisher Scientific), adjusted to an optical density (OD) of 0.05 at $600 \mathrm{~nm}$, and incubated for $15 \mathrm{~h}$ at $37^{\circ} \mathrm{C}$ with gentle agitation under microaerobic conditions.

The AGS human gastric epithelial cell line (ATCC CRL-1739) was maintained in Dulbecco's modified eagle medium (DMEM)/F-12 (Thermo Fisher Scientific) containing $10 \%(\mathrm{v} / \mathrm{v})$ FBS. AGS cells were seeded in six-well plates and grown to $\sim 80 \%$ confluence to be used for western blot analysis. For immunofluorescence microscopy, cells were seeded in six-well plates with cover glass and grown to $\sim 80 \%$ confluence $^{11}$. 
Antibodies and immunohistochemical reagents. The anti-Tyr(P)-CagA (antipY-CagA; for Western blot, 1:100; for immunofluorescence microscopy, 1:10), anti-UreA (for Western blot, 1:2000), and anti-VirB7 (for Western blot, 1:200) polyclonal antibodies were made from the antiserum of rabbits immunized with recombinant proteins or peptides ${ }^{11}$. Anti-CagA polyclonal antibodies (for Western blot, 1:1000) were purchased from AUSTRAL Biologicals (CA, USA), anti-actin monoclonal antibodies (for Western blot, 1:1000) were from MERCK (Darmstadt, Germany), horseradish peroxidase (HRP)-labeled anti-rabbit IgG (for Western blot, 1:100,000) and HRP-labeled anti-mouse IgG (for Western blot, 1:5000), and fluorescein isothiocyanate-labeled anti-rabbit IgG (for immunofluorescence microscopy, 1:100) was from Jackson ImmunoResearch Laboratories Inc. (PA, USA). DAPI (for staining, $300 \mathrm{nM}$ ) was from Sigma-Aldrich (MD, USA), and rhodamine-phalloidin (for staining, 1:100) was from Thermo Fisher Scientific (MA, USA).

Animal infection. H. pylori infection of rodents was performed as follows ${ }^{31,32}$ Briefly, 6-week-old male MON/Jms/GbsSlc Mongolian gerbils were orally administered $200 \mu \mathrm{L}$ Vancomycin $(500 \mathrm{mg} / \mathrm{L}) 24$ and $48 \mathrm{~h}$ before $H$. pylori inoculation. On the days of $H$. pylori inoculation, $300 \mu \mathrm{L} 5 \%(\mathrm{w} / \mathrm{v}$ ) sodium bicarbonate was orally administrated $10 \mathrm{~min}$ before bacterial inoculation. The gerbils were then intragastrically inoculated with an $\mathrm{H}$. pylori culture containing $10^{9}$ colony forming units (CFU) for 2 consecutive days. C57BL/6 mice (SLC Japan, Tokyo, Japan) were intragastrically inoculated once with $\mathrm{H}$. pylori culture of $10^{9} \mathrm{CFU}$. After the indicated date, the stomach of each infected animal was opened along the greater curvature. To quantitatively isolate $H$. pylori, the stomach was excised, weighed, and homogenized. Serial dilutions were plated on $\mathrm{H}$. pylori-selective agar plates (Eiken Chemical Co.) and incubated under microaerophilic conditions at $37^{\circ} \mathrm{C}$ for 4 days, after which the CFU were counted with a minimal detection limit of $1 \times$ $10^{3}$ CFU.

To isolate strains recovered from $H$. pylori-infected rodents, each colony on the $H$. pylori-selective agar plates was picked up by disposable inoculating loop and spread on Trypticase soy agar with $5 \%(\mathrm{v} / \mathrm{v})$ sheep's blood, and incubated under microaerophilic conditions at $37^{\circ} \mathrm{C}$ for two days. Then, colonies were suspended in Brucella broth supplemented with $5 \%(\mathrm{v} / \mathrm{v})$ inactivated FBS, adjusted to an OD of 0.05 at $600 \mathrm{~nm}$, and incubated for $15 \mathrm{~h}$ at $37^{\circ} \mathrm{C}$ with gentle agitation under microaerobic conditions. The cultures were preserved with $50 \%(\mathrm{v} / \mathrm{v})$ glycerol in $-80^{\circ} \mathrm{C}$ until use.

For RNA isolation, the tissue was immediately frozen in liquid nitrogen. All the animals were housed under controlled conditions with a $12 \mathrm{~h}$ light/dark cycle, $20-22{ }^{\circ} \mathrm{C}$ and $45 \pm 5 \%$ humidity. Animal experiments were conducted in accordance with the University of Tokyo or Osaka University guidelines for the care and use of laboratory animals and were approved by the Ethics Committee for Animal Experiments at the University of Tokyo or Osaka University.

Genomic DNA purification and sequencing. For PCR templates, genomic DNA was purified using InstaGene Matrix (Bio-Rad Laboratories, CA, USA).

For whole-genome sequencing, genomic DNA was purified from mid-log phase cultures of strain ATCC 43504 using QIAGEN DNeasy (QIAGEN). A genomic DNA library for sequencing was prepared using the Nextera XT DNA Sample Preparation kit (Illumina, San Diego, CA, USA) and sequenced using the Illumina MiSeq (for isolates from gerbils) or HiSeq X (for isolates from mice) platform to generate 300-bp paired-end reads. Genome assembly, scaffolding, and gap-closing were performed using the Platanus assembler ${ }^{33}$. Gene identification and annotation were conducted by the Microbial Genome Annotation Pipeline (MiGAP).

DNA sequences mutated in $>50 \%$ of the 40 strains recovered from Mongolian gerbils, or, in all of the 10 strains recovered from C57BL/6 mice are listed in Supplementary Data 2 and 4 . mRNA expression analysis was performed as follows: for genes in which the mutation occurred in the CDS region, mRNA expression of the CDS was measured; for mutations in the intergenic region, mRNA expression of an adjacent gene in which the intergenic region could be a $5^{\prime}$ UTR region was measured. HP1243 and HPG27_298, which started from the $3^{\prime}$ end of HP1243 with 33 nucleotides spaces, were regarded as a continuous gene since both genes are annotated as $b a b A$, and a ribosomal binding site (RBS) is assigned only at the upstream region of $\mathrm{HP} 1243^{34}$.

To confirm the number of T-repeats upstream of the HPnc4160 coding region, PCR was performed using primers designed to amplify around the region (Supplementary Data 8), and amplicons were purified by agarose gel electrophoresis and sequenced using an ABI3130xI DNA sequencer (Applied Biosystems, Foster City, CA, USA).

In vitro passage experiment. $H$. pylori ATCC 43504 was recovered from frozen stock and cultured on $5 \%(\mathrm{v} / \mathrm{v})$ sheep's blood agar for 2 days at $37^{\circ} \mathrm{C}$ in microaerobic conditions. Bacterial colonies were suspended in 3 tubes of Brucella broth supplemented with $5 \%(\mathrm{v} / \mathrm{v})$ inactivated FBS. Each bacterial suspension was adjusted to an $\mathrm{OD}$ of 0.05 at $600 \mathrm{~nm}$ and incubated for $12 \mathrm{~h}$ at $37^{\circ} \mathrm{C}$ with gentle agitation under microaerobic conditions. Subsequently, each fraction of the suspension was preserved by freezing in $50 \%(\mathrm{v} / \mathrm{v})$ glycerol as the "Original" strains. Meanwhile, each bacterial suspension was sub-cultured by resuspending in Brucella broth supplemented with $5 \%(\mathrm{v} / \mathrm{v})$ inactivated FBS adjusted to an OD of 0.05 at $600 \mathrm{~nm}$, and incubated an additional $12 \mathrm{~h}$ at $37^{\circ} \mathrm{C}$ with gentle agitation under microaerobic conditions. Sub-cultivation was repeated for 60 passages (30 days). Then, each cell suspension was preserved by freezing in $50 \%(\mathrm{v} / \mathrm{v})$ glycerol as the "60-passaged" strains. The "Original" and "60-passaged" strains were recovered from frozen stock on $5 \%(\mathrm{v} / \mathrm{v})$ sheep's blood agar by 2 days of incubation under microaerobic conditions. Then, colonies were suspended in Brucella broth supplemented with $5 \%(\mathrm{v} / \mathrm{v})$ inactivated FBS and incubated $12 \mathrm{~h}$ at $37^{\circ} \mathrm{C}$ with gentle agitation under microaerobic conditions. The bacterial cells were collected and subjected to genomic DNA purification.

RT-PCR. To prepare total RNA from H. pylori, liquid $H$. pylori cultures were agitated under microaerobic conditions at $37^{\circ} \mathrm{C}$ overnight until the $\mathrm{OD}$ value at $600 \mathrm{~nm}$ reached 0.9 .

Total RNA was extracted using ISOGEN (Nippon Gene, Tokyo, Japan), according to the manufacturer's instructions. The concentration of purified total RNA was analyzed using a NanoDrop Spectrophotometer (ThermoFisher Scientific, Wilmington, DE, USA). Total RNA was reverse transcribed into cDNA with a miScript II RT Kit (QIAGEN) according to the manufacturer's instruction ${ }^{35}$. mRNA expression was quantified and normalized to that of 23SrRNA (for H. pylori) or Gapdh (for mice and human) expression with THUNDERBIRD SYBR qPCR (TOYOBO) using the primer pairs described in Supplementary Data $8^{36}$. The results are expressed as the means \pm SEM from triplicate strain experiments.

Northern blot analysis. $2 \mu \mathrm{g}$ of total RNA in RNA loading dye (NEB) was separated by gel electrophoresis on $6 \%$ polyacrylamide/7 M urea gels in $1 \mathrm{xTBE}$ buffer for $3 \mathrm{~h}$ at $250 \mathrm{~V}$ and was electroblotted onto Hybond-XL nylon membrane (GE Healthcare) for $1 \mathrm{~h}$ at $50 \mathrm{~V}$ using Biometra Eco-Maxi system (Analytik-Jena). The membrane was crosslinked by $120 \mathrm{~mJ} / \mathrm{cm}^{2}$ UV light. Oligonucleotides JVO2624 and JVO-0485 to detect HPnc4160 and 5 S rRNA, respectively ${ }^{9}$, were $5^{\prime}$-endlabeled with $\left[{ }^{32} \mathrm{P}\right]-\gamma$-ATP by T4 polynucleotide kinase (Nippon Gene) and purified over G25 columns (GE Healthcare). After prehybridization in Rapid-Hyb buffer (Amersham), the $\left[{ }^{32} \mathrm{P}\right]$-labeled probe was hybridized at $42^{\circ} \mathrm{C}$ overnight. Membrane was washed in three steps in $5 \mathrm{x}$ SSC/0.1\% SDS, $1 \mathrm{x}$ SSC/0.1\% SDS and $0.5 \mathrm{x}$ SSC $/ 0.1 \%$ SDS buffers for $15 \mathrm{~min}$ at $42{ }^{\circ} \mathrm{C}$. Signals were visualized on Typhoon FLA7000 scanner (GE Healthcare).

\section{Genetic manipulation}

Construction of plasmids for producing gene-deficient mutants. Isogenic gene null mutants derived from ATCC 43504 were constructed by insertional mutagenesis as follows ${ }^{37}$. Using the extracted $H$. pylori ATCC 43504 genome as a template, DNA fragments containing the $500 \mathrm{bp}$ upstream region and the $500 \mathrm{bp}$ downstream region of the target gene were amplified by PCR using the primers (CagA KO up XhoI, CagA KO up EcoRI, CagA KO down BamHICagA KO down NotI, HPnc4160/HPnc4170 KO up KpnI, HPnc4160/4170 KO up ClaI, HPnc4160/ HPnc4170 KO down BamHI, HPnc4160/HPnc4170 KO down SacI) listed in Supplementary Data 8 . The DNA fragments were introduced at both sides of aphA3 (which confers kanamycin resistance) in pBluescript II SK (+) plasmids. The fragments from the resulting plasmids were introduced into $H$. pylori by electroporation.

Construction of non-marker H. pylori mutants. To construct non-marker H. pylori mutants, ATCC 43504 flaA and cag1 promoters and terminators were cloned into pBluescript SK(+ ) SmaI aphA3 SmaI, and $s a c B$ was cloned into the EcoRI site (pKSB plasmid).

Mid-log-phase (OD600 $=0.5-0.7)$ of $H$. pylori in $20 \mathrm{ml}$ liquid culture were washed twice with ice-cold $10 \%$ glycerol and resuspended by $200 \mu \mathrm{L}$ ice-cold $10 \%$ glycerol. One microgram of pKSB vector containing the target mutation and the bacterial cells were mixed at $4{ }^{\circ} \mathrm{C}$ and electroporated by a Micropulser (Bio-Rad) at the Ec2 $(2.5 \mathrm{kV})$ setting. After $4 \mathrm{~h}$ of incubation at $37^{\circ} \mathrm{C}$ in a microaerophilic condition, cells were plated on $5 \%$ sheep's blood agar TSAII plates containing $4 \mu \mathrm{g} / \mathrm{mL}$ kanamycin and incubated $2-3$ days at $37^{\circ} \mathrm{C}$ under microaerophilic conditions ${ }^{38}$. Four single colonies were seeded onto new $5 \%$ sheep's blood agar TSAII plates supplemented with $4 \mu \mathrm{g} / \mathrm{mL}$ kanamycin and incubated for an additional 2 days. Each colony was picked up and cultured in Brucella broth containing $5 \% \mathrm{FBS}$ at $37^{\circ} \mathrm{C}$ under microaerophilic conditions until $H$. pylori reached the mid-log phase. The medium $(100 \mu \mathrm{L})$ was plated on $5 \%$ sheep's blood agar plates supplemented with $2.5 \%$ sucrose and cultured for 2 days. Each colony was then seeded onto a new 5\% sheep's blood agar plate without antibiotics and incubated for 2 days. At the same time, colonies were also seeded onto a different agar plate with $4 \mu \mathrm{g} / \mathrm{ml}$ kanamycin to confirm that kanamycin resistance had been abolished. Surviving H. pylori were then transferred to liquid culture, and the genome sequence was confirmed by Sanger sequencing.

Construction of point-mutated H. pylori. The recombinant plasmids to establish various mutant T15, T16, T17, T18, and T19 H. pylori strains in the upstream region of hpnc4160 were constructed by PCR using genomic H. pylori DNA from the strains isolated from gerbils after 8 weeks as a template (primers: pKSBHPnc4160 Point mut ApaI and pKSB- HPnc4160 Point mut XhoI; listed in 
Supplementary Data 8), then, the resulting DNA fragments were cloned into suicide $\mathrm{pKSB}$ plasmids. To construct the recombinant plasmids to establish various mutant $H$. pylori strains in the upstream region of $h p n c 4160$ (T0, T1, T2, T3, T4, T5, T6, T7, T8, T9, T10, T11, T12, T13, and T20), mutated DNA fragments were constructed using PrimeSTAR Mutagenesis Basal Kit (Takara, Shiga, Japan) (template: pKSB HPnc4160 T15 plasmid; primers: T0-repT_s and T0-repT_as, T1repT_s and T1-repT_as, T3-repT_s and T3-repT_as, T4-repT_s and T4-repT_as, T5-repT_s and T5-repT_as, T6-repT_s and T6-repT_as, T7-repT_s and T7repT_as, T8-repT_s and T8-repT_as, T9-repT_s and T9-repT_as, T10-repT_s and T10-repT as, T11-repT_s and T11-repT_as, T12-repT_s and T12-repT_as, T13repT_s and T13-repT_as, T20-repT_s and T20-repT_as; listed in Supplementary Table 8). H. pylori T0, T1, T2, T3, T4, T5, T6, T7, T8, T9, T10, T11, T12, T13, T15, T16, T17, T18, T19, and T20 mutants were established by independently introducing the pKSB-based plasmids into the H. pylori ATCC 43504 strain.

Construction of NB-cagA-expressing H. pylori. Based on the full-length cagA cDNA sequence of ATCC 43504, we designed a HPnc4160-unbound cagA gene sequence (NB-cagA; Supplementary Fig. 5f, g). The NB-cagA cDNA was artificially synthesized as 908 bp pEX-K4J2-cagA mutants (Eurofins, 99900008281-1). cDNA fragments containing the mutated cagA sequence were amplified (primers: pKSBcagA-NB-ApaI, pKSB-cagA-NB-XhoI, listed in Supplementary Data 8), and cloned into suicide pKSB plasmids. The resulting plasmids were introduced to $H$. pylori ATCC 43504 to obtain NB-cagA-expressing H. pylori.

Construction of hpnc4160 over-expressing H. pylori. Plasmids for overexpression of hpnc4160 in H. pylori were constructed by combining DNA fragments of $h p n c 4160$ regions amplified by PCR using the primers pHel2- HPnc4160-de-4170-hed-f XhoI and pHel2- HPnc4160/HPnc4170-de-4170-hed-r BamHI (Supplementary Data 8) and genome DNA of the ATCC 43504 strain as a template. The resulting DNA fragments included the upstream region of $h p n c 4160$, excluding the $5^{\prime}$ region of the hpnc4170 region. The DNA was cloned into a pHel2 shuttle vector ${ }^{39}$ and introduced into $H$. pylori by electroporation.

RNA-seq. $H$. pylori were agitated under aerobic conditions and cultured at $37^{\circ} \mathrm{C}$ overnight until the $\mathrm{OD}$ value at $600 \mathrm{~nm}$ reached 0.9 . Total RNA from $H$. pylori was extracted using RNeasy (QIAGEN) following the manufacturer's instructions. The concentration of total RNA extracted was examined using a NanoDrop Spectrophotometer (ThermoFisher Scientific, Wilmington, DE, USA), according to the manufacturer's instructions.

Ten micrograms from each total RNA sample were treated with the MICROBExpress Bacterial mRNA Enrichment kit (Ambion, Grand Island, NY, USA) and RiboMinus ${ }^{\text {tx }}$ Transcriptome Isolation Kit (Bacteria) (Invitrogen, Grand Island, NY, USA) following the manufacturer's instructions. Samples were resuspended in $15 \mu \mathrm{L}$ RNase-free water. Bacterial mRNAs were chemically fragmented to 200-250 bp using $1 \times$ fragmentation solution (Ambion, Grand Island, NY, USA) for $2.5 \mathrm{~min}$ at $94^{\circ} \mathrm{C}$. cDNA was generated according to the instructions given in a SuperScript Double-Stranded cDNA Synthesis Kit (Invitrogen, Grand Island, NY, USA). Briefly, each mRNA sample was mixed with $100 \mathrm{pmol}$ random hexamers, incubated at $65^{\circ} \mathrm{C}$ for $5 \mathrm{~min}$, chilled on ice, mixed with $4 \mu \mathrm{L}$ First-Strand Reaction Buffer (Invitrogen, Grand Island, NY, USA), $2 \mu \mathrm{L}$ $0.1 \mathrm{M}$ DTT, $1 \mu \mathrm{L} 10 \mathrm{mM}$ RNase-freed NTPmix, $1 \mu \mathrm{L}$ SuperScript III reverse transcriptase (Invitrogen), and incubated at $50^{\circ} \mathrm{C}$ for $1 \mathrm{~h}$. To generate the second strand, the following Invitrogen reagents were added: $51.5 \mu \mathrm{L}$ RNase-free water, $20 \mu \mathrm{L}$ second-strand reaction buffer, $2.5 \mu \mathrm{L} 10 \mathrm{mM}$ RNase-free dNTP mix, $50 \mathrm{U}$ E. coli DNA Polymerase, $5 \mathrm{U}$ E. coli RNase $\mathrm{H}$, and incubated at $16^{\circ} \mathrm{C}$ for $2.5 \mathrm{~h}$. The Illumina Paired-End Sample Prep kit was used for RNA-seq library creation according to the manufacturer's instructions as follows: fragmented cDNA was end-repaired, ligated to Illumina adaptors, and amplified by 18 cycles of PCR. Paired-end 150-bp reads were generated by high-throughput sequencing with an Illumina Hiseq 2500 Genome Analyzer. After removing the low-quality reads and adaptors, RNA-seq reads were aligned to the corresponding ATCC 43504 genome using Tophat 2.1.0 $0^{40}$, allowing for a maximum of two mismatches. If reads mapped to more than one location, only that with the highest score was kept. Reads mapping to rRNA and tRNA regions were removed from further analysis. After obtaining read numbers from every sample, edgeR with TMM normalization method was used to determine differentially expressed genes. Significantly differentially expressed genes (FDR value $<0.05$ and at least two-fold change) were selected for further analysis ${ }^{41}$.

iTRAQ. H. pylori ATCC 43504 strains of wild-type, $\Delta h p n c 4160-h p n c 4170$, and $\Delta h p n c 4160$-hpnc4170 / pHel2-hpnc4160 were cultured in Brucella broth containing $5 \%$ fetal calf serum (FCS) to OD600 $=0.9$. Each bacterial solution $(1.5 \mathrm{~mL})$ was centrifuged at $5000 \times \mathrm{g}$ for $10 \mathrm{~min}$ at $4{ }^{\circ} \mathrm{C}$. The resulting pellet was resuspended in wash buffer ( $1 \mathrm{M} \mathrm{KCl}, 15 \mathrm{mM}$ Tris- $\mathrm{HCl}, \mathrm{pH}$ 7.4), centrifuged again, and the supernatant was removed ${ }^{42}$. The pellet was resuspended in a Wash buffer containing $1 \mathrm{mM}$ AEBSF (4- (2-Aminoethyl) benzenesulfonyl fluoride hydrochloride) and frozen at $-80^{\circ} \mathrm{C}$. iTRAQ analysis was commissioned to Filgen Corporation. Data were analyzed using ProteinPilot software (SCIEX, USA). Total ProtScore is a measure of the total amount of evidence for a detected protein and calculated using all of the peptides detected for the proteins. P values are measures of the certainty that the average ratio differs between samples.

EMSA (electrophoretic mobility shift assay). cDNA fragments of the entire small RNA hpnc4160 region; 150-bp fragments of the 5'UTR regions [from 100 bases upstream of the ribosome binding region (RBS), to 50 bases downstream of the RBS] of $h p 0410$ gene, $h p 0486$ gene, horB gene, $h p 0671$ gene, $h o p E$ gene, cagA gene, $h p 1227$ gene, and helpy_1262 gene; and 459 bp of the total cDNA containing the four hpnc4160-binding regions near the 3'tail of cagA, were amplified by PCR (primers: Small RNA HPnc4160 XhoI, Small RNA HPnc4160 EcoRI; HP0410 150 bp XhoI, HP0410 150 bp EcoRI; HELPY_0660 150 bp XhoI, HELPY_0660 150 bp EcoRI; HP0671 150 bp XhoI, HP0671 150 bp EcoRI; HP0486 150 bp XhoI, HP0486 150 bp EcoRI; HPSH_00635 150 bp XhoI, HPSH_00635 150 bp EcoRI; HPP12_0555 150 bp XhoI, HPP12_0555 150 bp EcoRI; HP1227 150 bp XhoI, HP1227 150 bp EcoRI; HELPY_1262 150 bp XhoI, HELPY_1262 150 bp EcoRI; CagA-B codding XhoI, CagA-B codding EcoRI; listed in Supplementary Data 8) and the ATCC 43504 genome as a template. The PCR products were cloned into the pBluescript SK $(+)$ plasmid downstream of the T7 promoter region. pBluescript SK (+) plasmid containing HPnc4160 mut (compensatory form for NBcagA mutation) was generated according to the instruction of PrimeSTAR Mutagenesis Basal Kit (Takara) using pBluescript SK $(+) h p n c 4160$ as a template (primers: HPnc4160_pointmut_primer_f, HPnc4160_pointmut_primer_r; listed in Supplementary Data 8). The NB-cagA mutant RNA used in the gel shift assay was amplified with a T7 promoter by PCR (T7 promoter CagA-NB EMSA PCR s, T7 promoter CagA-NB EMSA PCR as) and synthesized pEX-K4J2-CagA mutant (Eurofins, 99900008281-1) cagA as a template. cagA mutant RNA was prepared in the same manner except without mutations in the four HPnc4160-binding regions. RNA was transcribed from a DNA fragment using an in vitro Transcription T7 kit (Takara) ${ }^{43}$.

Gel shift assays were performed using 0.04 pmol 3'-biotin-tagged mRNA with increasing amounts of purified small RNA HPnc4160 in $20 \mu \mathrm{L}$ reactions. Briefly, RNA was denatured $\left(10 \mathrm{~min}, 80^{\circ} \mathrm{C}\right)$ and cooled for $5 \mathrm{~min}$ on ice. Yeast tRNA $(1 \mu \mathrm{g})$ (ThermoFisher SCIENTIFIC) was added to the labeled RNA, and binding buffer (10 mM HEPES pH 7.3, $1 \mathrm{mM} \mathrm{MgCl} 2,20 \mathrm{mM} \mathrm{KCl}, 5 \%$ glycerol) was added to a final volume of $10 \mu \mathrm{L}^{44}$. Then, $10 \mu \mathrm{L}$ labeled mRNA was added to HPnc4160. The mixtures were incubated at room temperature for $20 \mathrm{~min}$. Then the samples were mixed with $5 \mu \mathrm{L}$ native loading buffer before loading on a pre-cooled native $6 \%$ poly-acrylamide (PAA), $0.5 \times$ TBE gel. Gels were run in $0.5 \times$ TBE buffer at $30 \mathrm{~mA}$ per gel for $2 h^{45,46}$.

Cleavage assays. The cDNA of 720 bps of $H$. pylori rnase III was amplified by PCR (primers: pGEX-6P-1 RNaseIII XhoI-f, pGEX-6P-1 RNaseIII NotI-r, listed in Supplementary Data 8; and template: genome DNA from ATCC 43504 strain). The cDNA was cloned into a pGEX6P-1 vector (GE). E. coli BL21 transformed with the plasmids were subjected to shaking culture in LB broth containing $100 \mu \mathrm{g} / \mathrm{mL}$ ampicillin at $37^{\circ} \mathrm{C}$ with constant shaking at 200 r.p.m. Protein expression was induced with IPTG to a final concentration of $0.1 \mathrm{mM}$, at $4{ }^{\circ} \mathrm{C}$, for $4 \mathrm{~h}$. The bacteria were collected by centrifugation, and pellets were subjected to GST-fusion protein purification using Glutathione Sepharose 4B (GE) according to the manufacture's instruction. RNase III protein was excised by PreScission Protease according to the manufacturer's instructions. The purified protein derived from $6.7 \mathrm{~mL}$ of bacterial culture was developed by SDS-PAGE, and the gel was stained with Coomassie Brilliant Blue to confirm that no contaminants were observed in the final product. The protein concentration was determined by absorbance at $280 \mathrm{~nm}^{44}$.

Nuclease assays using RNase III were performed using purified $H$. pylori recombinant RNase III. The gel shift assay protocol described above was followed, except that an RNase III-specific buffer $(25 \mathrm{mM}$ Tris pH 7.5, $50 \mathrm{mM} \mathrm{NaCl}, 50 \mathrm{mM}$ $\mathrm{KCl}, 10 \mathrm{mM} \mathrm{MgCl} 2,1 \mathrm{mM}$ DTT) was used instead of Binding Buffer. 3 '-biotintagged partial cagA mRNA was incubated on ice with $5 \mu \mathrm{M}$ of small RNA HPnc4160 for $20 \mathrm{~min}$. RNase III was then added at a final concentration of $300 \mathrm{nM}$, and the reactions were incubated for $1 \mathrm{~min}$ at $37^{\circ} \mathrm{C}$. The samples were mixed with $5 \mu \mathrm{L}$ native loading buffer before loading on a pre-cooled native $6 \%$ PAA, 0.5x TBE gel ${ }^{47}$.

ELISA. AGS cells were co-incubated with $H$. pylori at a multiplicity of infection (MOI) of 100 for 12,24 and $36 \mathrm{~h}$ at $37^{\circ} \mathrm{C}$ in a $5 \% \mathrm{CO}_{2}$ environment in 24 well plates. The supernatants were collected and stored at $-30^{\circ} \mathrm{C}$. Enzyme-linked immunosorbent assays (ELISAs) for human IL-8 were performed using the Human IL-8 ELISA Kit (ThermoFisher SCIENTIFIC) according to the manufacturer's instructions. The results are expressed as the means \pm SEM from triplicate experiments.

Immunofluorescence microscopy. AGS cells were infected with $H$. pylori at an MOI of 100 for $6 \mathrm{~h}$ at $37^{\circ} \mathrm{C}$ in a $5 \% \mathrm{CO}_{2}$ environment. The cells were fixed with $4 \%$ $(\mathrm{w} / \mathrm{v})$ paraformaldehyde-PBS at room temperature for $10 \mathrm{~min}$. The cells were then washed with TBS 3 times, and blocked with Saponin buffer [10\% (v/v) Blocking One (Nakalai, Japan) containing $0.2 \%(\mathrm{w} / \mathrm{v})$ saponin] at $4{ }^{\circ} \mathrm{C}$ for $60 \mathrm{~min}$. Antibodies and fluorescent stains used for staining were DAPI, rhodamine-phalloidin (Thermo Fisher SCIENTIFIC, MA, USA), and anti-pY-CagA ${ }^{11,28}$. Confocal laser 
scanning microscopy (CLSM) image acquisition was performed using a Zeiss LSM 800 confocal laser scanning microscope with ZEN 2.3 software (Carl Zeiss, Jena, Germany)

Reporting summary. Further information on research design is available in the Nature Research Reporting Summary linked to this article.

\section{Data availability}

The raw read sequences and assembled scaffold sequences of $H$. pylori strains recovered from rodents or clinical isolates used in this study are available in the DDBJ/EMBL/Genbank databases under Bioproject accession numbers SAMD00178897 to SAMD00178935, SAMD00179460, SAMD00178937 and SAMD00204457 to SAMD00204466. Sequence data of the clinical isolates used in this study are available in the DDBJ/EMBL/Genbank databases with the accession codes listed in Supplementary Data 7. The authors declare that all other data supporting the findings of this study are available within the paper and its supplementary files. Source data are provided with this paper.

Received: 25 December 2020; Accepted: 10 March 2021; Published online: 09 April 2021

\section{References}

1. Hatakeyama, M. Helicobacter pylori CagA and gastric cancer: a paradigm for hit-and-run carcinogenesis. Cell Host Microbe 15, 306-316 (2014).

2. Kuipers, E. J., Perez-Perez, G. I., Meuwissen, S. G. M. \& Blaser, M. J. Helicobacter pylori and atrophic gastritis: importance of the cagA Status. J. Natl Cancer Inst. 87, 1777-1780 (1995).

3. Blaser, M. J. et al. Infection with Helicobacter pylori strains possessing cagA is associated with an increased risk of developing adenocarcinoma of the stomach. Cancer Res. 55, 2111-2115 (1995).

4. Suerbaum, S. \& Josenhans, C. Helicobacter pylori evolution and phenotypic diversification in a changing host. Nat. Rev. Microbiol. 5, 441-452 (2007).

5. Linz, B. et al. A mutation burst during the acute phase of Helicobacter pylori infection in humans and rhesus macaques. Nat. Commun. 5, 4165 (2014)

6. Kennemann, L. et al. Helicobacter pylori genome evolution during human infection. Proc. Natl Acad. Sci. USA 108, 5033-5038 (2011).

7. Yamaoka, Y. et al. Helicobacter pylori outer membrane proteins and gastroduodenal disease. Gut 55, 775-781 (2006).

8. Morelli, G. et al. Microevolution of Helicobacter pylori during prolonged infection of single hosts and within families. Plos Genet. 6, el001036 (2010).

9. Sharma, C. M. et al. The primary transcriptome of the major human pathogen Helicobacter pylori. Nature 464, 250-255 (2010).

10. Pernitzsch, S. R., Tirier, S. M., Beier, D. \& Sharma, C. M. A variable homopolymeric G-repeat defines small RNA-mediated posttranscriptional regulation of a chemotaxis receptor in Helicobacter pylori. Proc. Natl Acad. Sci. USA 111, E501 E510 (2014).

11. Mimuro, H. et al. Helicobacter pylori dampens gut epithelial self-renewal by inhibiting apoptosis, a bacterial strategy to enhance colonization of the stomach. Cell Host Microbe 2, 250-263 (2007).

12. Vannini, A. et al. Comprehensive mapping of the Helicobacter pylori NikR regulon provides new insights in bacterial nickel responses. Sci. Rep. 7, 45458 (2017).

13. Pernitzsch, S. R. \& Sharma, C. M. Transcriptome complexity and riboregulation in the human pathogen Helicobacter pylori. Front. Cell Infect. Microbiol. 2, 14 (2012).

14. Olejniczak, M. \& Storz, G. ProQ/FinO-domain proteins: another ubiquitous family of RNA matchmakers? New family of RNA matchmakers. Mol. Microbiol. 104, 905-915 (2017).

15. Wagner, E. G. H. \& Romby, P. Small RNAs in bacteria and archaea: who they are, what they do, and how they do it. Adv. Genet. 90, 133-208 (2015).

16. Hör, J., Matera, G., Vogel, J., Gottesman, S. \& Storz, G. Trans-acting small RNAs and their effects on gene expression in Escherichia coli and Salmonella enterica. Ecosal. Plus https://doi.org/10.1128/ecosalplus.ESP-0030-2019 (2020).

17. Aït-Bara, S. \& Carpousis, A. J. RNA degradosomes in bacteria and chloroplasts: classification, distribution and evolution of RNase E homologs: RNA degradosome evolution. Mol. Microbiol. 97, 1021-1135 (2015).

18. Odenbreit, S. et al. Translocation of Helicobacter pylori CagA into gastric epithelial cells by type IV secretion. Science 287, 1497-1500 (2000).

19. Mueller, D. et al. c-Src and c-Abl kinases control hierarchic phosphorylation and function of the CagA effector protein in Western and East Asian Helicobacter pylori strains. J. Clin. Invest. 122, 1553-1566 (2012)

20. Segal, E. D., Cha, J., Lo, J., Falkow, S. \& Tompkins, L. S. Altered states: Involvement of phosphorylated CagA in the induction of host cellular growth changes by Helicobacter pylori. Proc. Natl Acad. Sci. USA 96, 14559-14564 (1999).
21. Mimuro, H. et al. Grb2 is a key mediator of Helicobacter pylori CagA protein activities. Mol. Cell 10, 745-755 (2002).

22. Backert, S. \& Naumann, M. What a disorder: proinflammatory signaling pathways induced by Helicobacter pylori. Trends Microbiol. 18, 479-486 (2010).

23. Salaün, L., Ayraud, S. \& Saunders, N. J. Phase variation mediated niche adaptation during prolonged experimental murine infection with Helicobacter pylori. Microbiology 151, 917-923 (2005).

24. Salaün, L., Linz, B., Suerbaum, S. \& Saunders, N. J. The diversity within an expanded and redefined repertoire of phase-variable genes in Helicobacter pylori. Microbiology 150, 817-830 (2004).

25. Åberg, A. et al. A repetitive DNA element regulates expression of the Helicobacter pylori sialic acid binding adhesin by a rheostat-like mechanism. Plos Pathog. 10, 20 (2014).

26. Deitsch, K. W., Lukehart, S. A. \& Stringer, J. R. Common strategies for antigenic variation by bacterial, fungal and protozoan pathogens. Nat. Rev. Microbiol. 7, 493-503 (2009).

27. Saadat, I. et al. Helicobacter pylori CagA targets PAR1/MARK kinase to disrupt epithelial cell polarity. Nature 447, 330-333 (2007).

28. Suzuki, M. et al. Helicobacter pylori CagA phosphorylation-independent function in epithelial proliferation and inflammation. Cell Host Microbe 5, 23-34 (2009)

29. Eisenbart, S. K. et al. A repeat-associated small RNA controls the major virulence factors of Helicobacter pylori. Mol. Cell 80, 210-226.e7 (2020).

30. Hirukawa, S. et al. Characterization of morphological conversion of Helicobacter pylori under anaerobic conditions. Microbiol Immunol. 62, 221-228 (2018).

31. Kinoshita-Daitoku, R. et al. Mutational diversity in mutY deficient Helicobacter pylori and its effect on adaptation to the gastric environment. Biochem. Biophys. Res. 525, 806-811 (2020).

32. Satoh-Takayama, N. et al. 2 innate lymphoid cells in the stomach provide immune protection through induction of IgA. Immunity 52, 635-649.e4 (2020).

33. Kajitani, R. et al. Efficient de novo assembly of highly heterozygous genomes from whole-genome shotgun short reads. Genome Res. 24 1384-1395 (2014)

34. Yamaoka, Y. Roles of Helicobacter pylori BabA in gastroduodenal pathogenesis. World J. Gastroenterol. 14, 4265-4272 (2008).

35. Kiga, K. et al. Epigenetic silencing of miR-210 increases the proliferation of gastric epithelium during chronic Helicobacter pylori infection. Nat. Commun. 5, 4497 (2014).

36. Sanada, T. et al. The Shigella flexneri effector OspI deamidates UBC13 to dampen the inflammatory response. Nature 483, 623-626 (2012).

37. Skouloubris, S., Thiberge, J. M., Labigne, A. \& Reuse, H. D. The Helicobacter pylori UreI protein is not involved in urease activity but is essential for bacterial survival in vivo. Infect. Immun. 66, 4517-4521 (1998).

38. Donahue, J. P., Israel, D. A., Peek, R. M., Blaser, M. J. \& Miller, G. G. Overcoming the restriction barrier to plasmid transformation of Helicobacter pylori. Mol. Microbiol. 37, 1066-1074 (2000).

39. Heuermann, D. \& Haas, R. A stable shuttle vector system for efficient genetic complementation of Helicobacter pylori strains by transformation and conjugation. Mol. Gen. Genet. 257, 519-528 (1998).

40. Trapnell, C., Pachter, L. \& Salzberg, S. L. TopHat: discovering splice junctions with RNA-Seq. Bioinform. Oxf. Engl. 25, 1105-1111 (2009).

41. Fang, Y. et al. Transcriptome analysis of Xanthomonas oryzae pv. oryzicola exposed to $\mathrm{H} 2 \mathrm{O} 2$ reveals horizontal gene transfer contributes to its oxidative stress response. Plos ONE 14, e0218844 (2019).

42. Ariyoshi, M. et al. D-Glutamate is metabolized in the heart mitochondria. Sci. Rep. 7, 43911 (2017).

43. Mikami, M., Toki, S. \& Endo, M. In planta processing of the SpCas9-gRNA complex. Plant Cell Physiol. 58, 1857-1867 (2017).

44. Zhang, J. et al. Excessive miR-25-3p maturation via N6-methyladenosine stimulated by cigarette smoke promotes pancreatic cancer progression. Nat. Commun. 10, 1858 (2019).

45. Sim, S.-H. et al. Escherichia coli ribonuclease III activity is downregulated by osmotic stress: consequences for the degradation of bdm mRNA in biofilm formation. Mol. Microbiol. 75, 413-425 (2009).

46. Dugar, G. et al. The CsrA-FliW network controls polar localization of the dual-function flagellin mRNA in Campylobacter jejuni. Nat. Commun. 7, 11667 (2016).

47. Michaux, C. et al. RNA target profiles direct the discovery of virulence functions for the cold-shock proteins CspC and CspE. Proc. Natl Acad. Sci. USA 114, 6824-6829 (2017)

\section{Acknowledgements}

The authors would like to thank Manuel Amieva for providing the H. pylori strains. We acknowledge Keisuke Katsura and Junko Akada for their support. We would like to thank the members of the Division of Bacteriology, Department of Infectious Diseases 
Control, International Research Center for Infectious Diseases, The Institute of Medical Science, The University of Tokyo, and the members of the Department of Infection Microbiology, Research Institute for Microbial Diseases, Osaka University. This work was supported in part by Grant-in-Aid for Scientific Research from the Ministry of Education, Culture, Sports, Science, and Technology of Japan [17K19551, 18K07127, 19 K22704 (to H.M.), 16K07083 (to T.S.), 17K14974 (to M.T.), 20K16244 (to R.K.-D.)], the Naito Foundation (to H.M.), the Tokyo Biochemical Research Foundation (to H.M. and P.S.), the Kao Foundation for Arts and Sciences (to K.K.), and the Smoking Research Foundation (to H.M.).

\section{Author contributions}

R.K.-D., K.K. and H.M. conceived the study, designed experiments, and wrote the manuscript; M.M. performed Northern blot analysis; R.O. performed part of the mouse experiments; Z.B. analyzed gene-expression data sets; Y.O., R.Y., K.N. and T.H. analyzed genome sequences; T.V.P. and Y.Y. provided clinical isolates and analyzed genome sequences; T.Sa., T.O., T.I., E.K., S.H., M.T., A.S., P.S., H.A., and T.Su. provided technical assistance with experiments; T.T.B., L.T.N., K.V.V., and D.Q.D.H. provided clinical isolates; H.M. supervised the study.

\section{Competing interests}

The authors declare no competing interests.

\section{Additional information}

Supplementary information The online version contains supplementary material available at https://doi.org/10.1038/s41467-021-22317-7.
Correspondence and requests for materials should be addressed to H.M.

Peer review information Nature Communications thanks Richard Peek Jr, Gisela Storz and the other, anonymous, reviewer for their contribution to the peer review of this work. Peer reviewer reports are available.

Reprints and permission information is available at http://www.nature.com/reprints

Publisher's note Springer Nature remains neutral with regard to jurisdictional claims in published maps and institutional affiliations.

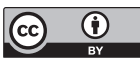

Open Access This article is licensed under a Creative Commons Attribution 4.0 International License, which permits use, sharing, adaptation, distribution and reproduction in any medium or format, as long as you give appropriate credit to the original author(s) and the source, provide a link to the Creative Commons license, and indicate if changes were made. The images or other third party material in this article are included in the article's Creative Commons license, unless indicated otherwise in a credit line to the material. If material is not included in the article's Creative Commons license and your intended use is not permitted by statutory regulation or exceeds the permitted use, you will need to obtain permission directly from the copyright holder. To view a copy of this license, visit http://creativecommons.org/ licenses/by/4.0/.

(C) The Author(s) 2021 